\title{
Validación de los Modelos de Cambio Climático hidrostáticos y no hidrostáticos sobre la climatología de Ecuador en las variables de precipitación y temperaturas extremas
}

\author{
Validation of hydrostatic and non-hydrostratic climate models on precipitation \\ variables and extreme temperatures on Ecuador's weather
}

\author{
Enrique Palacios Chacón* y Sheila Serrano Vincenti
}

Centro de Investigación en Modelamiento Ambiental CIMA, Universidad Politécnica Salesiana, Quito, Ecuador.

*Autor para correspondencia:wpalacios@ups.edu.ec

Artículo recibido el 28 de febrero de 201 I.Aprobado, tras revisión el 20 de abril de 201 I.

\begin{abstract}
Resumen
La presente investigación tiene como objetivo determinar las bondades o deficiencias de los modelos de cambio climático PRECIS hidrostático y TL959 no hidrostático frente a la climatología de Ecuador, basándose en la validación de la precipitación y temperaturas extremas. Se plantea la hipótesis de que si estos modelos de cambio climático reconstruyen adecuadamente la climatología mediante cada una de sus climatologías bases iniciales, entonces sus proyecciones hacia el futuro serán más ciertas. Con este objetivo, se dividió a Ecuador en una matriz II x II, permitiendo su estudio por regiones, donde se determinaron estadísticos para cada celda mes a mes, tanto para la climatología como para los modelos. Al encontrar las diferencias de los estadísticos del modelo menos los de la climatología se estableció que es necesario mejorar la parametrización física en zonas motañosas de gran altura, típicas de la geografía andina del país, ya que en estas regiones se encontró el mayor desajuste de los modelos.
\end{abstract}

Palabras clave: modelos de cambio climático, PRECIS,TL959, climatología, Ecuador.

\begin{abstract}
The present research aims to determine the advantages and disadvantages of two climate change models. The hydrostatic model PRECIS and the non-hydrostatic model TL959 were used to validate precipitation and extreme temperatures on Ecuador's weather. The main hypothesis establishes that if the climate change model adequately reconstruct the climate through each of their initial climatology conditions, then their projections into the future will be more accurate. We divided the surface of Ecuador in a I |x I I matrix allowing a regional study and determined statistical estimators for each cell every month for both the weather and the models. The differences between the model and the climatology increase in highlands regions, suggesting an improvement of the physical parametrization in Andes regions.
\end{abstract}

Keywords: climate change models, PRECIS, TL959, climatology, Ecuador. 


\section{Introducción}

El contar con modelos de cambio climático validados es de suma importancia, tanto para las regiones de Ecuador como para el mundo en general ya que esta herramienta permite conocer con antelación la presencia de ciertos eventos de tipo climático como sequías, inundaciones y otros desastres naturales por condiciones meteorológicas extremas, éstas causarían menor daño si se conocieran con cierta anticipación. Para realizar esta tarea, se ha tomado el modelo climático regional anidado (RCM por sus siglas en inglés), el cual fue propuesto por primera vez por Dickinson et al. (1989), seguido por los trabajos de Giorgi y Bates (1989); paulatinamente, simulaciones de RCM de largo alcance han ido incrementándose hacia un amplio rango de aplicaciones, incluyendo validaciones, estudios de sensibilidad y aciertos sobre cambio climático, sobre este último punto existe una extensa revisión bibliográfica realizada por Giorgi \& Meanrns (1999) y XinZhong (2004).

En la actualidad, es comúnmente aceptado que un downscaling, es decir una reducción de escala de un RCM tiene mejores resultados para resolver los problemas generados por la orografía en clima que los modelos de circulación general sobre mallas cruzadas o GCM (por General Circulation Model en inglés), especialmente por la simulación de variables muy cercanas a la superficie terrestre. (Girogi, 1990; Jones et al., 1995; Giorgi et al., 1997, 1998; Laprise et al., 1998; Leung y Ghan, 1999; Hong y Leetmaa, 1999; Fennessy y Shukla, 2000; Pan et al. 200I y Roads et al. 2003). Sin embargo, existe un sesgo sistemático en los RCMs que no ha sido completamente explicado, y existen grandes dispersiones sobre regiones que no son lo suficientemente planas (Takle et al., 1999; Leung et al., 1999; Pan et al., 200I; Anderson et al., 2003). Los resultados de los RCM son sensibles a sus configuraciones dinámicas, como la resolución del dominio y las representaciones físicas (como, por ejemplo, la radiación sobre las nubes y las interacciones entre la superficie y la atmósfera). Por lo tanto, un RCM típico debe reducir al máximo cada aplicación regional específica (Giorgi y Mearns, 1999). Esto incluye experimentos cuidadosamente sensitivos y rigurosos estudios de validación, utilizando los datos iniciales observados y las condiciones adecuadas de frontera.

Bajo este contexto, se presenta un estudio del tratamiento de series de tiempo de las variables de precipitación y temperaturas extremas, que serán contrastadas con las salidas de los dos modelos PRE$\mathrm{CIS}$, (pronunciado de esta forma del francés, précis - "PRAY-sea"), modelo hidrostático desarrollado en el Reino Unido, y el modelo no hidrostático TL959 (modelo global de muy alta resolución, desarrollado en Japón). Este segundo modelo reproduce el clima del país, con salidas y condiciones de clima de 25 años, tomados de 105 estaciones meteorológicas a través del cálculo de la energía, identificando los valores y vectores propios-dado que la energía es la integral del trabajo- y los parámetros meteorológicos fueron tomados como matrices, cada región singular fue calculada con desviaciones de energía, las cuales fueron necesarias para identificar las áreas con desviaciones más intensas de energía y así poder ajustarse a las condiciones de tiempo.

\section{Materiales y métodos}

\section{I Datos y métodos}

En nuestro país existe una gran variabilidad en el comportamiento de la precipitación y de su ciclo anual, con significativos incrementos en la región media de la costa y disminuciones moderadas en la mitad de la región andina, justamente sobre la cordillera de los Andes de Ecuador. En la mayoría de lugares en donde existe un registro de las precipitaciones del país se ha verificado un incremento en la intensidad de la lluvia. Al igual que en la mayor parte de las estaciones se ha registrado un aumento de los valores de climas extremos, es decir que los máximos y mínimos se han exagerado.

Bajo este contexto, una tarea importante para la adecuada comprensión y manejo de los parámetros de los procesos dinámicos y físicos que ocurren en la región ecuatorial tropical es la de tener una climatología adecuada y compararla con la climatología genera- da por modelos de precisión (Control-Climatología), como la generada por el Modelo TL959 y el PRECIS (a los que llamaremos Alfa-Climatología) y compararla con la generada a partir de información de las estaciones meteorológicas. "Todos estos datos de propiedad" del INAMHI (Instituto Nacional de Meteorología e Hidrología) información que llamaremos Beta-Climatología. "Los datos utilizados fueron las salidas gráficas de los modelos de precisión los cuales fueron comparados con las salidas de las estaciones meteorológicas en tierra a través de los programas de procesamiento de imágenes de MatLab (poner sello de marca registrada), como se aprecia en la Figura I".

\subsection{Datos en PRECIS}

En primera instancia se discutirán los resultados que serán presentados y aplicados en este estudio, correspondientes al modelo PRECIS; este modelo utiliza una resolución de $25 \mathrm{~km}$ y, por tanto, existe una diferenciación entre la Costa, la cordillera de la Costa, la depresión central y los Andes. Es decir, que esta simulación, como un modelo climático regional, ofrece 
la información espacial con suficiente detalle. PRECIS es un modelo alimentado de un modelo global de la atmósfera HadAM3P, que es uno acoplado océano-atmósfera desarrollado en el Centro Hadley del Reino Unido.

El modelo global entrega en primer lugar las condiciones de frontera de la región que se utilizarán, que en nuestro caso incluyen el territorio continental del Ecuador. Es importante indicar que las observaciones del aumento de la temperatura superficial del mar fueron reproducidas con exactitud por el modelo global HadCM3. De esta manera, se obtienen las condiciones de borde precisas y actualizadas una vez al día, cada día hasta completar 30 años. Estas condiciones de frontera son necesarias para delimitar los dominios con los que inicializará el modelo (Jones et al., 2004).

Este modelo de componentes atmosféricos es una versión hidrostática de todas las ecuaciones primitivas que se trabajan en el estudio de la atmósfera, es decir, ésta se asume en el estado de equilibrio hidrostático y, por lo tanto, se diagnostican movimientos verticales separadamente de las ecuaciones de estado. Así se logra una representación completa de la fuerza de Coriolis y utiliza una malla regular de latitud y longitud en la horizontal y una coordenada vertical híbrida. Hay 19 niveles verticales, la mínima de 50 metros y la más alta de $0,5 \mathrm{hPa}$ con coordenadas sigma ( $\sigma=$ presión / presión en la superficie) utilizado por cuatro bajas, tres coordenadas puramente a la presión de la parte superior y las combinaciones entre ellos en los medios.

Las ecuaciones del modelo se resuelven en coordenadas esféricas y la red de longitud-latitud se rota de manera que el Ecuador se encuentra dentro de la región de interés a fin de obtener zonas prácticamente uniformes en toda la región. La resolución horizontal es $0,44^{\circ} \times 0,44^{\circ}$, lo que da una resolución mínima de aproximadamente $50 \mathrm{~km}$ en Ecuador de la cuadrícula rotada. Debido a esta alta resolución, el modelo requiere un intervalo de tiempo de 5 minutos para mantener la estabilidad de cómputo (Jones et al., 2004).

\subsection{Datos en TL959}

El segundo modelo que se utiliza en este estudio es considerado un modelo de alta resolución, conocido como TL959 desarrollado en Japón. Es un modelo de circulación atmosférica general, con una resolución de tamaño de la cuadrícula de $20 \mathrm{~km}$ en la horizontal; ha sido desarrollado por científicos japoneses haciendo uso de un Simulador Terrestre ES (Earth Simulator)que constituye un sistema de superordenadores vectoriales paralelos y consta de 5.120 procesadores, el cual fue posicionado como la computadora más rápida del mundo para realizar cálculos en el 2004. Su objetivo era obtener evidencias científicas de los posibles impactos del calentamiento global en fenómenos de pequeña escala, tales como ciclones tropicales y el frente baiu en el monzón de verano del este de Asia. El mismo modelo fue desarrollado para simular el clima real con alta precisión a través de mejoras en los esquemas de parametrización, los procesos físicos y las comparaciones con los datos observados. Esta tecnología funciona con un sistema de memoria distribuido en paralelo, que consta de 640 nodos de procesador, cada nodo es un sistema de memoria compartida que contiene 8 procesadores vectoriales.

La biblioteca MPI para la paralelización fue utilizada entre los nodos y se previeron microtareas de memoria compartida en paralelo. El cálculo de la eficiencia es del $30 \%$ de rendimiento máximo. El superordenador tendrá 4 horas para realizar una integración de un mes en el TL959L60 (redes horizontales de 20 $\mathrm{km}$ y 60 niveles verticales) con 30 nodos (240 CPUs) (Mizuta et al., 2005). Cabe indicar que no hay modelos climáticos globales que soporten las integraciones de largo plazo, mantener el clima global y generar simulaciones realistas de masas como fue diseñado en el Japón. Aunque $10 \mathrm{~km}$ es la resolución máxima que se utiliza en este país y que ha simulado con éxito los ciclones tropicales, ciclones extratropicales y problemas de crecimiento de frentes; el periodo de integración abarca un par de semanas. Integraciones de menor tiempo de los modelos globales con resolución más alta se realizan por diversos grupos como simulaciones del clima a largo plazo, de II años T239LI8 (50 millas), utilizando el modelo CCM3 del Centro Nacional de Investigaciones Atmosféricas NCAR (por sus siglas en inglés) en los EE.UU., también 12 años con T319L24 (40 km de resolución) del mismo centro de investigación.

\subsection{Climatología de Ecuador}

Llamada en este trabajo climatología beta, consiste en la información meteorológica real del país con una serie de registros históricos de 25 años; en Ecuador existen solamente cerca de 25 estaciones con una cobertura concentrada en el espacio central y sur de la región interandina, dejando grandes áreas sin registros en la Costa y Oriente del país y, por lo tanto, este primer conjunto de 25 se complementa con series de 15 y 10 años para cubrir el resto del país. Previamente se realizó un análisis de la representatividad de estas series históricas para incorporarlas en la climatología beta. Así, se obtiene un total de 105 estaciones meteorológicas que conforman la serie de tiempo en 
tiempo real o base, llamada climatología beta, además se incluye la realización de un análisis estadístico de consistencia antes de apoyar adecuadamente la serie.

Hay que tener en cuenta que las observaciones del INAMHI se comparan en tiempo real con las simulaciones de los modelos PRECIS y TL959 con el fin de verificar que el clima en un tiempo dado coincida con el generado por los modelos; las variables observadas fueron únicamente la precipitación y temperaturas extremas.

Como se puede observar, los dos modelos utilizados en este estudio tienen una malla de diferente resolución horizontal, PRECIS alcanza una resolución de $25 \mathrm{~km}$, mientras que el modelo TL959 llega a una resolución de $20 \mathrm{~km}$. Así, para los estudios se incluyó la distribución de red de malla teniendo todos la misma resolución de $20 \mathrm{~km}$, desde el análisis objetivo de Cressman (1959), con el fin de obtener una mejor comprensión sobre la distribución espacial de los parámetros meteorológicos.

De esta manera, se dividió al país como si éste fuera una matriz de II $\times$ II en el que las tres primeras columnas se hicieron corresponder a la Costa, las dos siguientes a la Sierra y desde la sexta en adelante se ubicó al Oriente de Ecuador, logrando estudiar separadamente a las regiones del país que tienen, sobre todo, características orográficas diferentes. A cada celda de esta matriz, se le hizo corresponder numéricamente los promedios mes a mes de las variables climáticas estudiadas en esta región durante 30 años. Bajo este criterio, se estudió, en primera instancia, a las estaciones meteorológicas con información de al menos 5 años; a continuación se incorporaron a esta lista las estaciones con al menos 10 años de datos meteorológicos y así de forma sucesiva hasta incluir aquellas con 30 años de información; abarcando de esta manera a todas las estaciones existentes en la red del INAMHI. En aquellos casos en los que existían vacíos de datos se realizó un relleno utilizando el mencionado análisis objetivo de Cressman.

Específicamente, esta investigación incluye el estudio de tres variables: la temperatura mínima, la máxima y la precipitación, los promedios de éstas, tomados mes a mes durante 30 años se ubican en cada celda.

\subsection{Diferencias entre los resultados de los modelos de cambio climático: PRECIS y TL959 vs. la climatología del Ecuador}

Después del procesamiento de las imágenes de los modelos y de la climatología pixel a pixel, a través de MatLab," se obtuvieron los datos numéricos de las variables estudiadas para cada región, delimitada por la matriz en que fue dividido el Ecuador; promediando estos datos mes a mes y para los 30 años, se lograron los estadísticos que representan el comportamiento de la precipitación y temperaturas extremas por mes; esta homogenización en el tipo de datos permitió realizar una comparación efectiva entre los datos simulados por los modelos y la climatología construida con datos reales.
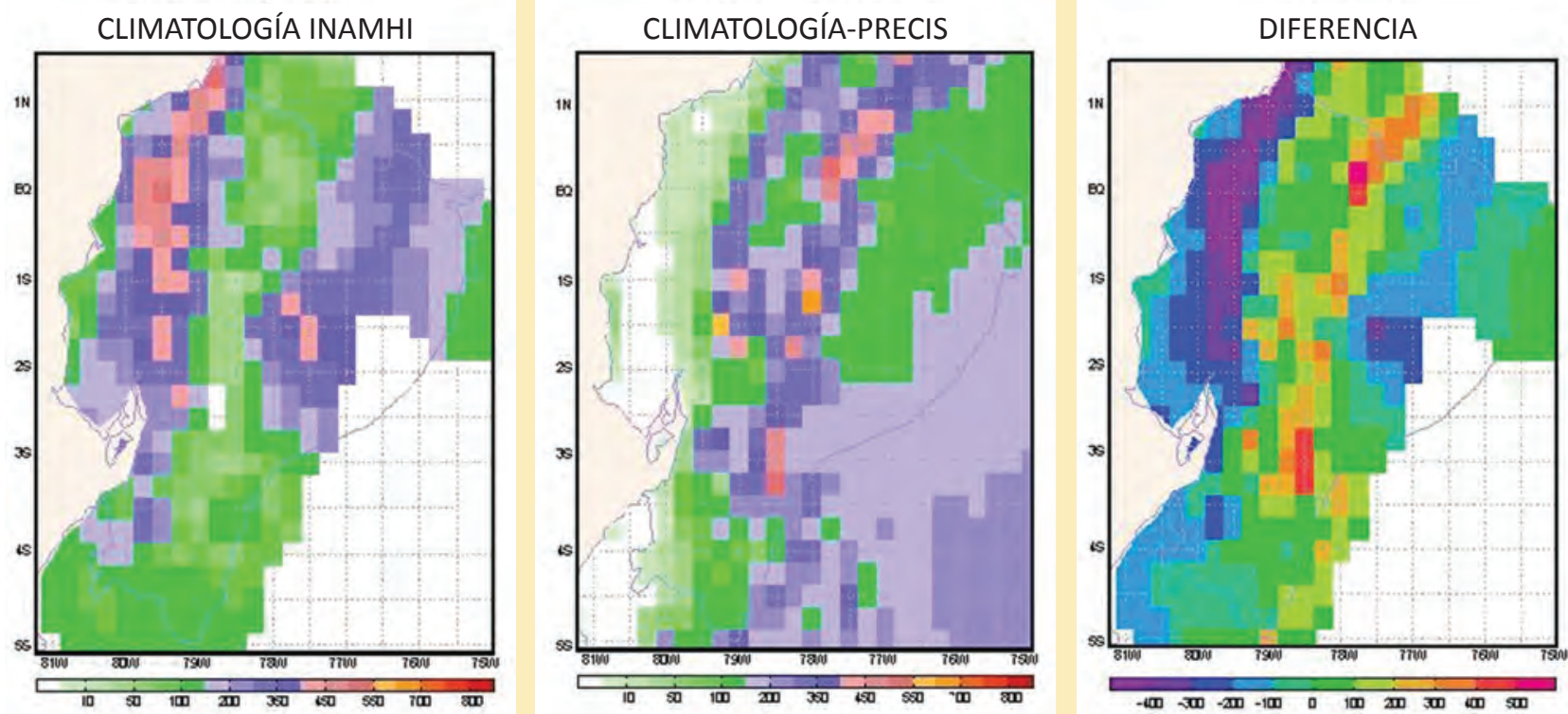

Figura 1. (a) Climatología lograda de las Series de Tiempo con datos del INAMHI para Ecuador para el mes de enero en la variable de precipitación. (b) Climatología lograda con el modelo PRECIS para el mes de enero en la variable de precipitación. (c) Diferencia de los valores de la climatología generada por el modelo menos la climatología de las series de tiempo, los valores en rojo representan sobreestimación y en café subestimación 

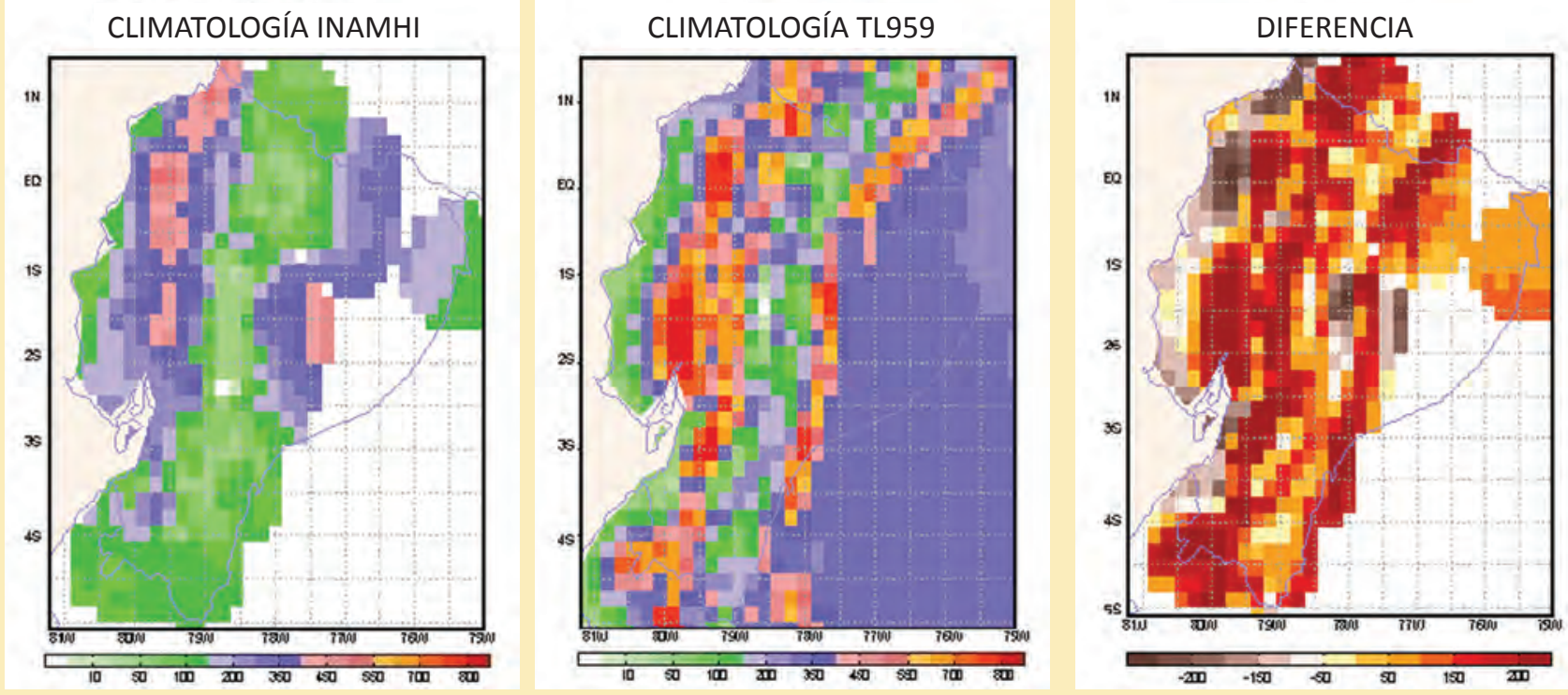

Figura 2. (a) Climatología lograda de las Series de Tiempo con datos del INAMHI para Ecuador para el mes de enero en la variable de precipitación. (b) Climatología lograda con el modelo TL959 para el mes de enero en la variable de precipitación. (c) Diferencia de los valores de la climatología generada por el modelo menos la climatología de las series de tiempo, los valores en rojo representan sobreestimación y en café subestimación.

Restando los valores numéricos entregados por cada uno de los modelos de los valores determinados de la climatología, celda por celda y para cada una de las tres variables meteorológicas se pudo conocer la efectividad entre PRECIS, TL959 y la Climatología de Ecuador (ver Figuras I y 2).

\section{Resultados}

\section{I Análisis de la diferencia de los valores temperatura mínima entregados por los modelos de cambio climático y la climatología del país, región por región}

Al analizar la región Costa el histograma de dispersión de la diferencia entre los valores de temperaturas mínimas entregadas por los modelos PRECIS y TL959 vs. la climatología de Ecuador, se puede apreciar que la gran mayoría de las diferencias son nulas (ver Figura 3, Tabla I). Asimismo se aprecia que el modelo PRECIS se ajusta mejor a la climatología, logrando un promedio bajo de tan solo $0,399^{\circ} \mathrm{C}$ de diferencia con la climatología con una desviación estándar de $1,354^{\circ} \mathrm{C}$; frente a los valores logrados con el modelo japonés TL959 en el que se tiene también un promedio de $0,916^{\circ} \mathrm{C}$ de diferencia con la climatología y una desviación estándar de $2,735^{\circ} \mathrm{C}$.
Revisando los diagramas de cajón; en PRECIS se nota que prácticamente no existen datos entre los cuartiles primero y tercero que sean diferentes de cero; lo cual indica una gran coincidencia entre lo modelado y lo real, aunque existen unos pocos casos en los que se puede decir que el modelo ha sobreestimado la temperatura mínima. Mientras que al analizar el diagrama de cajón del modelo TL959, se nota que la mayoría de datos se encuentran en el cuartil superior, esto quiere decir que también existe una sobreestimación de la temperatura mínima para este modelo.

En el caso de estudio sobre la Sierra de Ecuador, se aprecia que ambos modelos tienen sus máximos en cero, lo cual indica que en la mayoría de casos, no existe diferencia entre lo modelado y la climatología del país; sin embargo, sí se puede decir que estos datos están más dispersos que en la Costa. Para el modelo PRECIS, se tiene un promedio de diferencias de $1,453^{\circ} \mathrm{C}$ y una desviación estándar de $3,505^{\circ} \mathrm{C}$ que es significativa; estos valores aumentan al analizar al modelo TL959 en donde se tiene una media de $1,9^{\circ} \mathrm{C}$ de diferencia y una desviación estándar de $3,854^{\circ} \mathrm{C}$. Al analizar los diagramas de cajón, se aprecia que PRECIS acumula sus datos en la región superior del cuartil, lo que indica una sobrestimación elevada de la temperatura mínima, mientras que en TL959 esta sobreestimación es ligera, sin olvidar que proviene de datos más dispersos. 
Los modelos de cambio climático, se ajustan bastante bien a las condiciones en la región oriental, con un promedio de diferencias de $0,6949^{\circ} \mathrm{C}$ y una desviación de tan sólo $2,103^{\circ} \mathrm{C}$ para PRECIS, mientras que TL959 tiene un promedio de diferencias de $0,2639^{\circ} \mathrm{C}$ con una desviación ligeramente mayor de $2,768^{\circ} \mathrm{C}$ (ver Figura 3). Este buen ajuste del modelo, se evidencia también al analizar los diagramas de cajón, en donde se ve que los cuartiles están muy cercanos, lo cual indica una dispersión mínima de los datos; en este caso ambos modelos, aunque poco, sobreestiman ligeramente la temperatura mínima.

\subsection{Análisis de la diferencia de los valores temperatura máxima entregados por los modelos de cambio climático y la climatología del país, región por región}

Al analizar qué tan bien modela PRECIS a temperatura máxima en la región Costa, se encuentran un promedio de $1,23^{\circ} \mathrm{C}$ con una desviación de $2,36^{\circ} \mathrm{C}$ (ver Figura 4) más altos que para la temperatura mínima; analizando el diagrama de cajón, la diferencia de modelo menos climatología es positiva, ya que hay más datos en el cuar- til superior, lo que indica que PRECIS a sobreestimado también a la temperatura máxima. Mientras que TL959, presenta un promedio bastante bueno de $-0,2894^{\circ} \mathrm{C}$, pero con una considerable desviación de $3,885^{\circ} \mathrm{C}$. Analizando los diagramas de cajón, vemos que la dispersión es más o menos simétrica, con una ligera tendencia a la sobreestimación de la temperatura máxima. Con estos datos, se puede decir que la dispersión para PRECIS es mejor que para TL959.

Para el caso de estudio de la región Sierra, se encuentra que PRECIS tiene una media de diferencias de solo $-0,1312^{\circ} \mathrm{C}$, pero una dispersión de $4,364^{\circ} \mathrm{C}$, lo cual indica mayor dispersión que para el caso de la temperatura mínima, además, analizando los diagramas de cajón se nota una distribución mayoritaria en el cuartil inferior lo que indica una subestimación de este modelo sobre la temperatura máxima, a diferencia de lo que ocurría en la Costa. El comportamiento de TL959 es más disperso, con una media de $-2,903^{\circ} \mathrm{C}$ tiene una desviación de $4,413^{\circ} \mathrm{C}$ (ver Figura 4, Tabla I), el diagrama de cajón muestra una dispersión bastante simétrica, aunque elevada; esto quiere decir que en este caso no se ha sobreestimado o subestimado la temperatura máxima por este modelo.
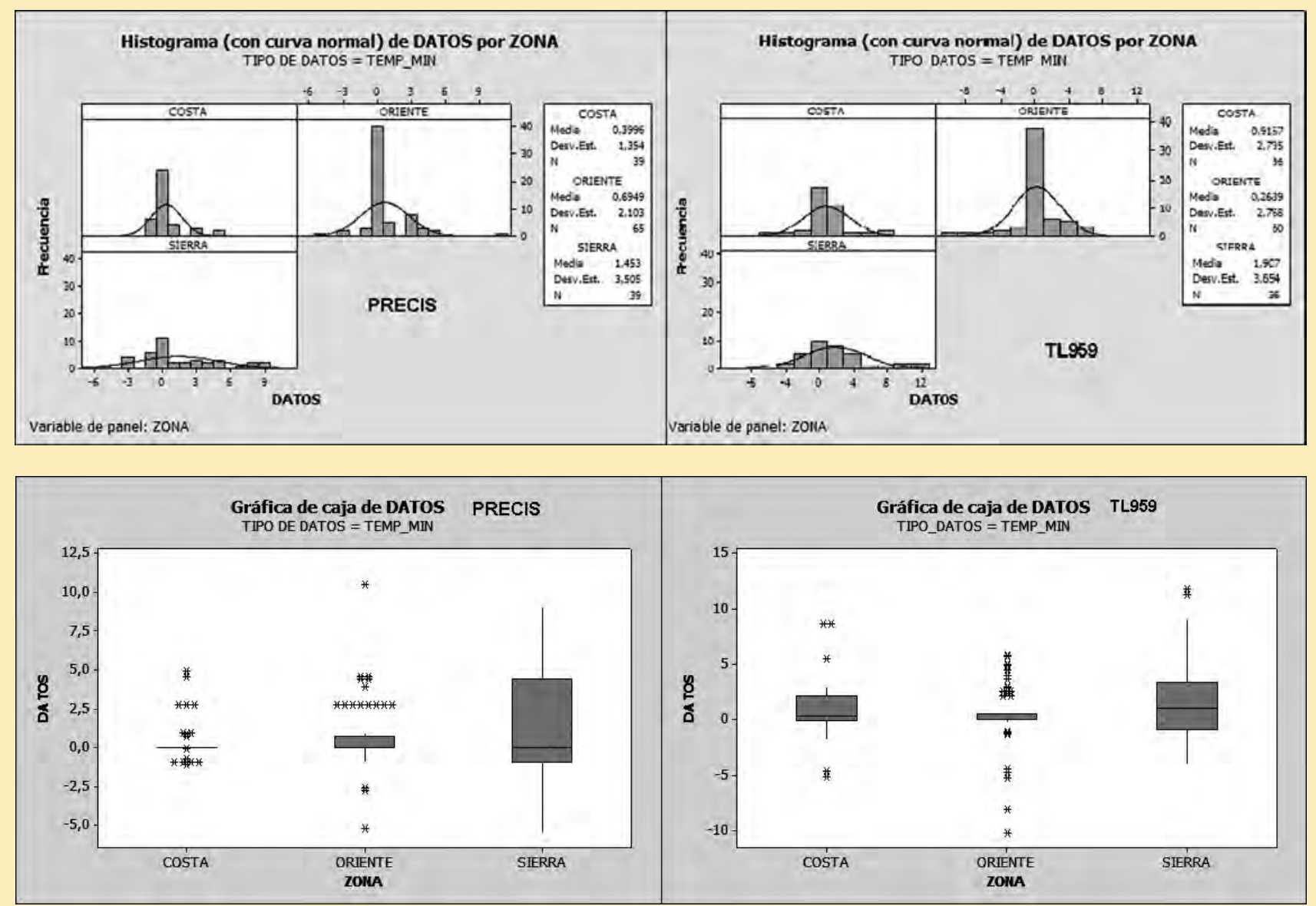

Figura 3. Histograma de frecuencias y diagramas de cajón de las diferencias entre los valores de temperaturas mínimas entregadas por los modelos PRECIS y TL959 menos los valores de la climatología para cada región del Ecuador. 

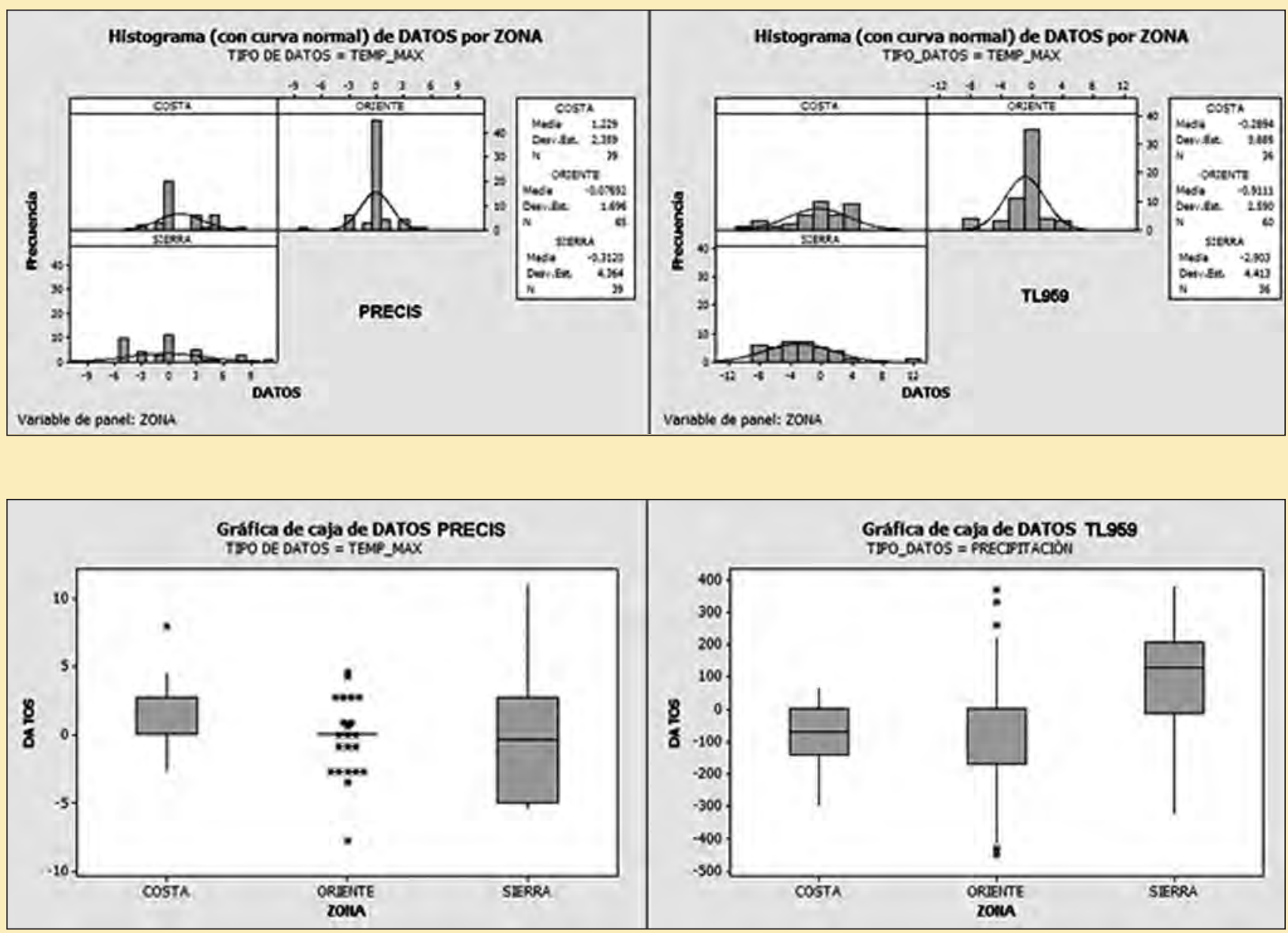

Figura 4. Histogramas de frecuencias y diagramas de cajón de las diferencias entre los valores de temperaturas máximas entregadas por los modelos PRECIS y TL959 menos los valores de la Climatología, para las diferentes regiones del Ecuador

Asimismo, al analizar las temperaturas máximas en el Oriente se encuentran diferencias promedio de $-0,0769^{\circ} \mathrm{C}$ con desviaciones de tan solo $1,696^{\circ} \mathrm{C}$ para PRECIS, y una media de diferencias $-0,9111^{\circ} \mathrm{C}$ y una desviación de $2,590^{\circ} \mathrm{C}$ para TL959. Estos ajustes en realidad son muy satisfactorios, más aún cuando se analizan los diagramas de cajón, al ver el comportamiento de PRECIS prácticamente no existen datos dentro del rango intercuartil; mientras que TL959 localiza datos mayoritariamente en el cuartil inferior, lo que nos lleva a pensar que subestima la temperatura máxima.

\subsection{Análisis de la diferencia de los valores entregados por los modelos de cambio climático y la climatología del país para la variable precipitación, en las diferentes regiones}

Al analizar el comportamiento en la costa de PRECIS, se visualizan diferencias promedios de $-108,5 \mathrm{~mm}$ con una desviación de 106,2 mm; mientras que a diferencia del comportamiento de TL959 muestra mejores ajustes: con una media de solo $-81,27 \mathrm{~mm}$ y una desviación de 9I,26 mm (ver Figura 5, Tabla I), si analizamos los diagramas de cajón, se aprecia que ambos modelos han subestimado la gran mayoría los valores de precipitación en la Costa.

En la región Sierra, los promedios de diferencias para la precipitación entregados por PRECIS son de $-56,4$ I mm y una desviación 166,5; mientras que TL959 logra una media de $102 \mathrm{~mm}$ y una desviación de $156,0 \mathrm{~mm}$. Los diagramas de cajón muestran una sobreestimación de los valores de precipitación.

En el Oriente se presenta un mejor comportamiento a todo nivel; PRECIS con promedios de -36,4I $\mathrm{mm}$ y una desviación III,4mm; mientras que TL959 logra una media de $-61,40 \mathrm{~mm}$ y una desviación de I66,0 mm. Los diagramas de cajón muestran una subestimación de los valores de precipitación para ambos modelos. 

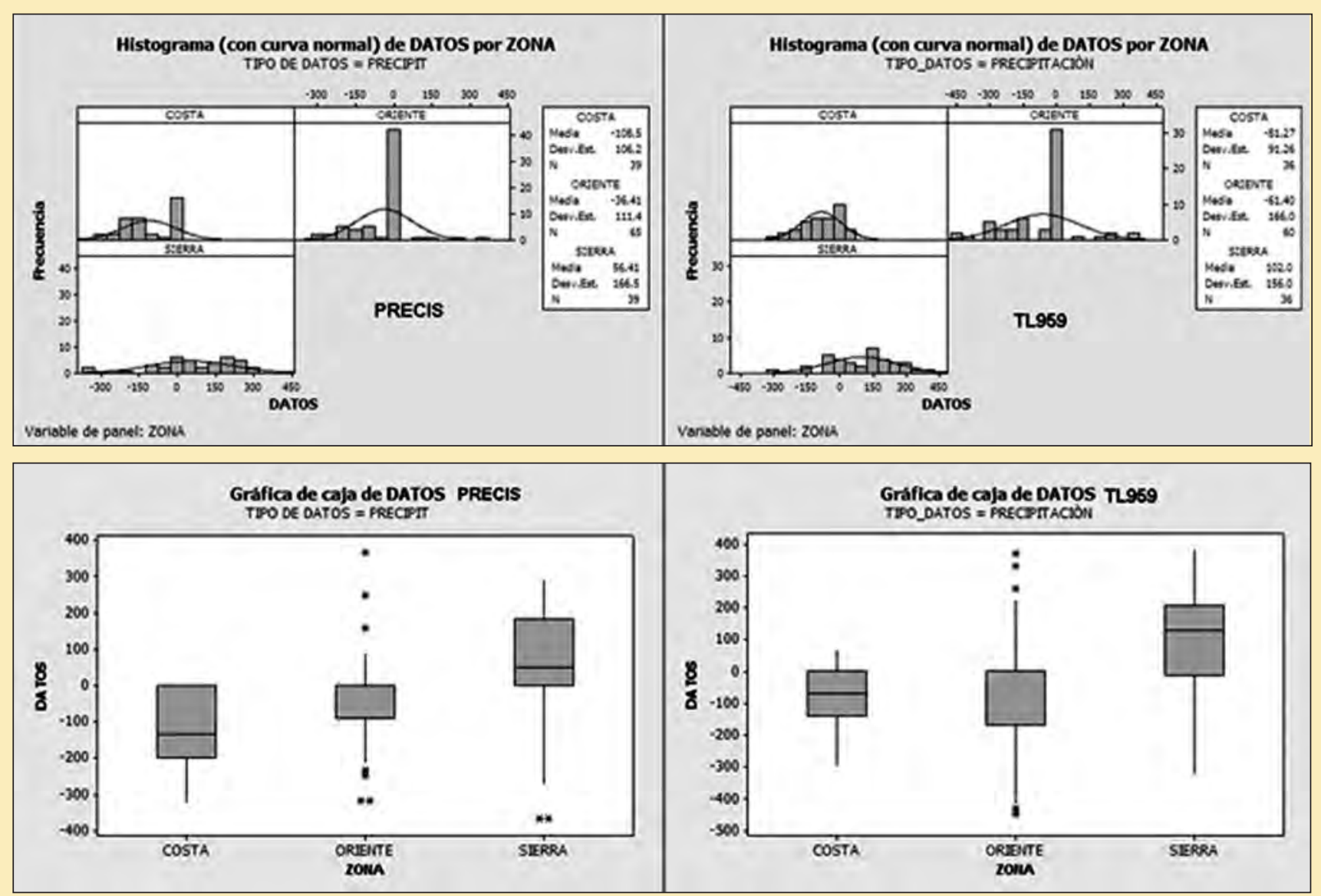

Figura 5. Histogramas de frecuencias y diagramas de cajón de las diferencias de los valores entregados por los modelos PRECIS y TL959 menos los de la climatología de las diferentes regiones de Ecuador para la precipitación

Tabla I. Medias y desviaciones estándar de las diferencias entre las climatologías de los modelos PRECIS y TL959 menos la climatología real de Ecuador, además se indica el caso en que existió sobreestimación o subestimación de los datos entregados por parte del modelo

\begin{tabular}{|c|c|c|c|c|c|c|}
\hline \multirow{2}{*}{ REGIÓN } & \multicolumn{2}{|c|}{ COSTA } & \multicolumn{2}{|c|}{ SIERRA } & \multicolumn{2}{|c|}{ ORIENTE } \\
\hline & PRECIS & TL959 & PRECIS & TL959 & PRECIS & TL959 \\
\hline T-MÍN $\left({ }^{\circ} \mathrm{C}\right)$ & $\begin{array}{c}\mu: 0,3996 \\
\sigma: 1,354 \\
\text { Sobrestimación }\end{array}$ & $\begin{array}{c}\mu: 0,9167 \\
\sigma: 1,354 \\
\text { Sobrestimación }\end{array}$ & $\begin{array}{c}\mu: 1,453 \\
\sigma: 3,505 \\
\text { Sobrestimación }\end{array}$ & $\begin{array}{c}\mu: 1,907 \\
\sigma: 3,854 \\
\text { Sobrestimación }\end{array}$ & $\begin{array}{c}\mu: 0,6949 \\
\sigma: 2,103 \\
\text { Sobrestimación }\end{array}$ & $\begin{array}{c}\mu: 0,2639 \\
\sigma: 2,768 \\
\text { Sobrestimación }\end{array}$ \\
\hline T- MÁX $\left({ }^{\circ} \mathrm{C}\right)$ & $\begin{array}{c}\mu: 1,229 \\
\sigma: 2,359 \\
\text { Sobrestimación }\end{array}$ & $\begin{array}{c}\mu:-0,2894 \\
\sigma: 3,885 \\
\text { Sobrestimación }\end{array}$ & $\begin{array}{c}\mu:-0,3120 \\
\sigma: 4,34364 \\
\text { Subestimación }\end{array}$ & $\begin{array}{c}\mu:-2,903 \\
\sigma: 4,413 \\
\text { Subestimación }\end{array}$ & $\begin{array}{c}\mu:-0,07692 \\
\sigma: 1,696\end{array}$ & $\begin{array}{c}\mu:-0,91 \mathrm{II} \\
\sigma: 2,590 \\
\text { Subestimación }\end{array}$ \\
\hline R.R (mm) & $\begin{array}{c}\mu:-108,5 \\
\sigma: 106,2 \\
\text { Subestimación }\end{array}$ & $\begin{array}{c}\mu:-81,27 \\
\sigma: 91,26 \\
\text { Subestimación }\end{array}$ & $\begin{array}{c}\mu: 56,41 \\
\sigma: 166,5 \\
\text { Sobrestimación }\end{array}$ & $\begin{array}{c}\mu: 102,0 \\
\sigma: 156,0 \\
\text { Sobrestimación }\end{array}$ & $\begin{array}{c}\mu:-36,4 \mid \\
\sigma:|| I, 4 \\
\text { Subestimación }\end{array}$ & $\begin{array}{c}\mu:-61,40 \\
\sigma: 166,0 \\
\text { Subestimación }\end{array}$ \\
\hline
\end{tabular}




\section{Conclusiones}

Las diferencias encontradas al restar los valores modelados por PRECIS y TL959, menos los de la climatología real de Ecuador, muestran que el comportamiento de la temperatura mínima fue sobreestimado por ambos modelos en las tres regiones, lo que quiere decir que las temperaturas mínimas reales fueron más extremas. El mejor ajuste se logró en la zona oriental donde prácticamente no existe región montañosa,y el peor en la región interandina aunque PRECIS, en general, presentó un mejor comportamiento que TL959. Asimismo, al analizar las temperaturas máximas, se evidencia también que el modelo inglés es superior al japonés, aunque ambos presentan el mismo estado de sobreestimación o subestimación por región. En este caso, ambos sobreestiman la temperatura máxima en la región Costa y la región oriental, presentándose la temperatura máxima real menor a lo modelado en estas zonas; mientras que en la región Sierra ésta es subestimada, registrándose realmente temperaturas más elevadas.

Sin embargo, es necesario apuntar que ambos modelos al trabajar con temperaturas extremas no se ajustan con suficiente exactitud según los estándares requeridos para el comportamiento climático en los trópicos y subtrópicos.

En cuanto al comportamiento de las diferencias entre el modelo y la climatología real en la variable de precipitación, los errores aumentan de manera inusual, al punto que no se puede determinar qué modelo se ajusta mejor; aunque sí existe un acuerdo en cuanto a la sobreestimación o subestimación por región, en este caso se ha subestimado la precipitación en la región Costa y oriental, en las que la precipitaciones registradas fueron mayores. Mientras, que en la región Sierra, la precipitación fue sobreestimada ya que los valores reales fueron mucho menores a los modelados.

Posiblemente, la razón para estos desajustes radique en el cálculo exagerado de los vientos provocados por la presencia de montañas y elevaciones, resultado de los pesos de los componentes en las ecuaciones diferenciales de ambos modelos. Lo cual se evidencia por el aumento del error en regiones montañosas tanto para el cálculo de las temperaturas extremas como par los valores de la precipitación; inclusive el exagerado error en el Modelamiento de la precipitación se deba a efectos excesivos de convección sobre las irregulares zonas montañosas del país. Se recomienda, entonces, mejorar la parametrización física en zonas montañosas de gran altura, típicas de la geografía andina del país, así como mejorar la resolución del estudio para incluir con más detalle las características físicas de la zona.

\section{Agradecimientos}

Los autores agradecen a las autoridades de la Universidad Politécnica Salesiana por el auspicio a la presente investigación. De igual manera al Professor Mizuta del JMC (Japan Meteorological Centre), por los datos suministrados del modelo TL959, sin los cuales este trabajo no habría sido posible.

\section{Referencias}

Anderson, C. J. Coauthors. 2003. Hydrological processes in regional climate model simulations of the central United States flood of June-July 1993. J. Hydrometeor 4:584-598.

Christensen, O. B., J. H. Christensen, B. Machenauer and M. Bozet. 1998. Very high-resolution regional climate simulations over Scandinavia-Present climate. J. Climate II:3204-3229.

Dickinson, R. E., R. M. Erroco, F. Giorgi and G.T. Bates. 1989. A regional climate model for the western United States. Climate Change 15: 383-422.

Fennessy, M. J. and J. Shukla. 2000. Seasonal prediction over North America with a regional model nested in a global model. J. Climate 13: 2605-2627.

Giorgi, F. 1990. On the simulation of regional climate using a limited area model nested in a general circulation model. J. Climate 3:94I-963.

Giorgi, F., J.W. Hurrell, M. R. Marinucci and M. Beniston. 1997. Elevation signal in surface climate change: A model study. J. Climate 10 : 288-296.

Giorgi, F., L. Mearns, C. Shields and L. McDaniel. 1998. Regional nested model simulations of present day and $2 \times \mathrm{CO} 2$ climate over the central Great Plains of the United States. Climate Change 40:457-493. 
Giorgi, F. and G.T. Bates. 1989. On the climatological skill of a regional model over complex terrain. Mon.Wea. Rev I 17:2325-2347.

Giorgi, F. and L. O. Mearns. 1999. Introduction to special section: Regional climate modeling revisited. J. Geophys. Res 104: 6335-6352.

Giorgi, F. 1990. On the simulation of regional climate using a limited area model nested in a general circulation model. J. Climate 3:94I-963.

Giorgi, F. and L. O. Mearns. 1999. Introduction to special section: Regional climate modeling revisited. J. Geophys. Res. 104: 6335-6352.

Hong, S-Y. and A. Leetmaa. 1999. An evaluation of the NCEP RSM for regional climate modeling. J. Climate 12:592-609.

Jones, R. G., J. M. Murphy and M. Noguer. 1995. Simulation of climate change over Europe using a nested regional climate model, I,Assessment of control climate, including sensitivity to location of lateral boundary conditions. Quart. J. Roy. Meteor. Soc I21: |4|3-|449.

Jones, S., M. Jones, and S. Deo. Using keyphrases as search result surrogates on small screen devices. Personal and Ubiquitous Computing, 8(I): 55-68, 2004.
Takle, E. S. Coauthors. 1999. Project to intercompare regional climate simulations (PIRCS): Description and initial results. J. Geophys. Res 104: 19443-1946I.

Leung, L. R., S. J. Ghan, Z-C. Zhao, Y. Luo, W-C. Wang and H-L. Wei. 1999. Intercomparison of regional climate simulations of the 1991 summer monsoon in eastern Asia. J. Geophys. Res 104:6425-6454.

Laprise, R., D. Caya, M. Giguere, G. Berger, J. P. Blanchet, G. J. Boer and N. A. McFarlane. 1998. Climate and climate change in on, $\mathbf{H}$. Cote western Canada as simulated by the Canadian regional climate model. Atmos.-Ocean 36: I19-167.

Pan, Z., J. H. Christensen, R.W.Arritt,W.J. Gutowski Jr., E. S. Takle and F. Otieno. 200I. Evaluation of uncertainties in regional climate change simulations. J. Geophys. Res. 106: |7735-|775|.

Roads, J., S-C. Chen and M. Kanamitsu. 2003. U.S. regional climate simulations and seasonal forecasts. J. Geophys. Res., 108, 8606, doi:I0.1029/2002JD002232

Xin-Zhong Liang, Li Li and Kenneth E. Kunkel. 2004. Regional Climate Model Simulation of U.S. Precipitation during 1982-2002. Part I: Annual Cycle. Journal of Climate 2004; 17: 3510-3529.

\section{Fe de erratas:}

Página 22, segunda columna, al final del tercer párrafo dice:

[...] las estaciones meteorológicas del INAMHI (Instituto Nacional de Meteorología e Hidrología) información que llamaremos Beta-Climatología.

Y debe decir:

[...] las estaciones meteorológicas. "Todos estos datos de propiedad" del INAMHI (Instituto Nacional de Meteorología e Hidrología) información que llamaremos Beta-Climatología. "Los datos utilizados fueron las salidas gráficas de los modelos de circulación los cuales fueron comparados con las salidas de las estaciones meteorológicas en tierra a través de los programas de procesamiento de imágenes de MATLAB $®$, como se aprecia en la Figura I".

En la página 24, segunda columna, tercer párrafo dice:

Una vez corridos los modelos, se obtuvieron los datos numéricos de las variables estudiadas para cada región,...

Debe decir:

"Después del procesamiento de las imágenes de los modelos y de la climatología pixel a pixel, a través de MATLABß," se obtuvieron los datos numéricos de las variables estudiadas para cada región,... 